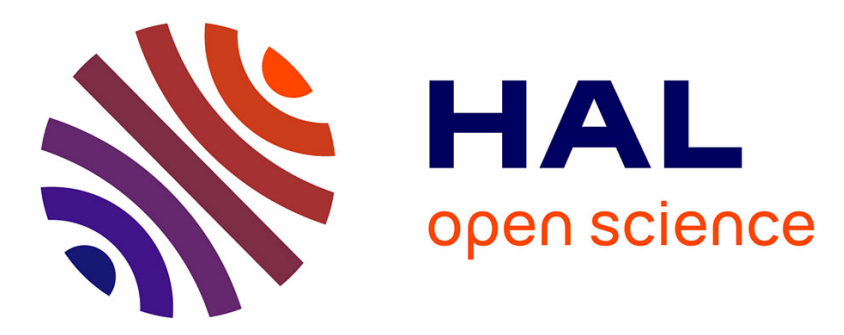

\title{
Field-To-Long-Segmented-Trace Coupling with Arbitrary Loads and a Transparent Upper Bound Using a Single Modified Taylor Cell
}

Sjoerd Op 'T Land, Mohamed Ramdani, Richard Perdriau, M'Hamed Drissi, Frank Leferink

\section{To cite this version:}

Sjoerd Op 'T Land, Mohamed Ramdani, Richard Perdriau, M'Hamed Drissi, Frank Leferink. FieldTo-Long-Segmented-Trace Coupling with Arbitrary Loads and a Transparent Upper Bound Using a Single Modified Taylor Cell. IEEE Transactions on Electromagnetic Compatibility, 2016, 58 (5), pp.1517-1525. 10.1109/TEMC.2016.2566449 . hal-01377005v2

\section{HAL Id: hal-01377005 \\ https://hal.science/hal-01377005v2}

Submitted on 6 Oct 2016

HAL is a multi-disciplinary open access archive for the deposit and dissemination of scientific research documents, whether they are published or not. The documents may come from teaching and research institutions in France or abroad, or from public or private research centers.
L'archive ouverte pluridisciplinaire HAL, est destinée au dépôt et à la diffusion de documents scientifiques de niveau recherche, publiés ou non, émanant des établissements d'enseignement et de recherche français ou étrangers, des laboratoires publics ou privés. 


\title{
Field-To-Long-Segmented-Trace Coupling with Arbitrary Loads and a Transparent Upper Bound Using a Single Modified Taylor Cell
}

\author{
Sjoerd Op 't Land ${ }^{* \dagger}$, Student Member, IEEE, Mohamed Ramdani ${ }^{* \dagger}$, Senior Member, IEEE, \\ Richard Perdriau* ${ }^{* \dagger}$, Senior Member, IEEE \\ $\mathrm{M}^{\prime}$ hamed Drissi ${ }^{\dagger}$, Senior Member, IEEE, Frank Leferink ${ }^{\ddagger}$, Senior Member, IEEE \\ ${ }^{*}$ ESEO-EMC, ESEO-TECH, \\ Angers, France \\ Email: \{sjoerd.optland, mohamed.ramdani, richard.perdriau\}@eseo.fr \\ ${ }^{\dagger}$ ADH-SRC, IETR \\ Rennes, France \\ Email: mhamed.drissi@insa-rennes.fr \\ Telecommunication Engineering Group, Faculty of EEMCS, University of Twente, The Netherlands \\ Email: f.b.j.leferink@utwente.nl
}

\begin{abstract}
In modern electronic products, the printed circuit board (PCB) traces may well form the dominant coupling path in radiated immunity problems. Therefore, an understanding of the designable parameters that influence the worst-case induced voltages can be of use to the PCB designer, together with rapid simulations.

Therefore, a modified single (unmeshed) Taylor cell is combined with transmission line theory to predict the terminal voltages induced by a grazing, vertically polarized plane wave, incident on a multi-segment trace with arbitrary terminal impedances. The resulting model is closed-form and therefore suitable for rapid simulations. Furthermore, the model is geometrically approximated to provide understanding on how designable PCB parameters determine the worst-case induced voltage. Finally, the model is compared to measurement results.
\end{abstract}

Index Terms-field-to-trace coupling, modified Taylor model, closed-form solution, full-wave simulation, GTEM cell

\section{INTRODUCTION}

In modern, unshielded wireless electronics, PCB traces are amongst the largest metallic structures. Therefore, they may well be the major contributors to a coupling path for incident electromagnetic disturbances. Simple predictive models for field-to-trace coupling could therefore be of use to the EMCaware PCB designer.

To clearly discuss the quality of these models, some terminology will first be introduced. Using this terminology, the state of the art will be evaluated. Then, the gap this article tries to close will be presented. Finally, the structure of the article remainder will be outlined.

\section{A. Transparent Models}

Engineers have a number of degrees of freedom to solve a problem: the design parameters (e.g. resistor value, trace length or circuit topology). These design parameters span the design space $D$, which contains every possible design. The

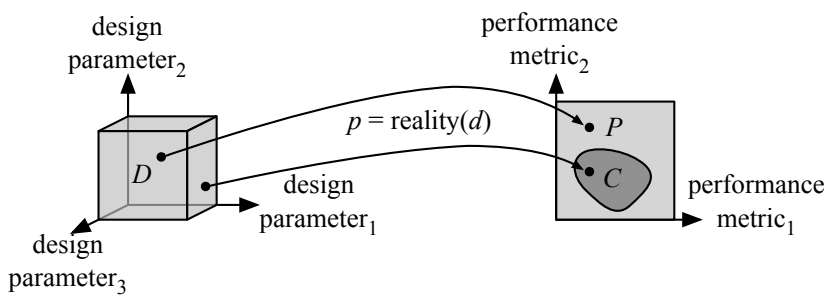

Figure 1. Reality maps design space into performance space. One successful design and one unsuccessful design are shown.

problem is solved when the performance of the design complies with the requirements, expressed in terms of performance metrics (e.g. immunity, speed, bandwidth, cost or development time). These performance metrics span the performance space $P$, of which compliance $C$ is a subspace (e.g. immunity meets or exceeds DO-160F and development time is shorter than one month). Reality determines the performance of each design candidate, reality : $D \rightarrow P$. To successfully design is to find a design, of which the performance $p$ is compliant with the requirements: any $d \in D$, such that $\operatorname{reality}(d) \in C$, as illustrated by Figure 1.

As the dimension of design space is generally high and reality is too hard to understand, let alone invertible, simplified models are always employed. One modeling technique is design-oriented approximation: reality is approximated to become easily invertible. For example, a resistive voltage divider is required to have a voltage transfer $H=1 / 3$, when loaded by an unknown load $R_{L}>10 \mathrm{k} \Omega$. The real relation between design parameters $\left(R_{1}\right.$ and $\left.R_{2}\right)$ and the performance metric $(H)$ is given by

$$
H=\frac{R_{1} / / R_{L}}{R_{1} / / R_{L}+R_{2}}=\frac{R_{1} R_{L}}{R_{1} R_{2}+R_{1} R_{L}+R_{2} R_{L}} .
$$

Suppose that $R_{L}$ and $R_{1}\left(R_{2}\right)$ would be given, it is not obvious 
to find $R_{2}\left(R_{1}\right)$. The equation is true, but one cannot 'see through' it, the relation is opaque [1].

Conversely, when $R_{1}$ is chosen much smaller than $R_{L}$, the transfer becomes approximately:

$$
H \approx \frac{R_{1}}{R_{1}+R_{2}} \rightarrow \frac{R_{2}}{R_{1}} \approx \frac{1}{H}-1,
$$

and $R_{2}$ follows naturally. Moreover, one sees directly that only the ratio between $R_{1}$ and $R_{2}$ is fixed by $H$. Hence, the relation is considered transparent.

To summarize, the relation between design parameters and performance metrics can become more transparent by approximation. The price paid is reduction in accuracy and in validity domain (above approximation only holds for $R_{1} \ll R_{L}$ ).

Note that transparency is subjective: experienced engineers will also see through (1). However, even these subjects will consider (2) more transparent than (1). Therefore, relative transparency is objective.

\section{B. State of the Art}

There are currently three equivalent formulations of field-tobifilar transmission line coupling: [2]: that of Taylor et al. [3], Agrawal et al. [4] and that of Rachidi [5]. These formulations suppose weak coupling, i.e. no interaction from transmission line to incident field is considered. The coupling is modeled with distributed voltage and/or current sources along the line. In particular cases, the resulting terminal voltages can be cast in a closed form, for example using the Baum-Liu-Tesche (BLT) equation [6]. Generally, however, the terminal voltages must be obtained using a circuit simulator.

Paul has very well elaborated the coupling of an arbitrary polarized, arbitrary incident field to a lossy, multi-conductor transmission line (MTL) in a homogeneous medium [7]. However, a PCB trace is not homogeneous, and his analysis does not apply in the general case. Nevertheless, Paul's solution served as a cross-validation of the analysis presented in the present article [8].

Leone has studied the case of an arbitrary polarized, arbitrary incident plane wave to a single microstrip segment [9]. Based on Agrawal's formulation and the BLT equation, he found a closed-form, opaque solution for arbitrary loads and a transparent solution for characteristic loads, showing that the latter solution is a reasonable approximation for moderately mismatched loads. Moreover, in this approximation, he showed that the low-frequency worst case voltage occurs at the near-end terminal for endfire illumination.

Based on Leone's opaque solution, Lagos et al. have studied the worst-case induced voltage on a single microstrip segment, by means of fast numerical optimisation [10]. However, this method only yields a result for particular loads and a particular frequency, and no general insight is obtained: the method is opaque. Vanhee et al. accelerated load optimisation for an MTL line with a given geometry [11]. They only perform the time-consuming finite elements simulation once, with open terminations. With the results, they can quickly calculate the worst case induced voltages or currents for any set of terminations. Therefore, this method is interesting for a designer who optimizes MTL terminations, but not for the PCB designer who optimizes routing, because the latter keeps modifying the geometry. Moreover, because it involves finite element simulation, the results are opaque. Magdowski explored the typical-case coupling of a randomly incident, randomly polarized field to a homogeneous transmission line with arbitrary loads [12]. His results are closed-form, but only transparent for electrically short lines with particular loads.

Approaching industrial reality, Leone has successfully applied the equivalent wire method and the Method of Moments (MoM) to quickly simulate the coupling to a multi-segment, branched trace with mismatched loads [13]. As his method relies on numerical simulation, it is opaque as well.

In order to gain understanding, Op 't Land started to develop a transparent upper bound for the coupling of a grazing, vertically polarized plane wave to a microstrip with characteristic loads [14]. This model is transparent, but somewhat limited with regards to real-life PCB traces.

\section{Contribution}

In order to understand real-life coupling problems, the limitations (elevation, polarization and termination) should be removed one by one, without losing transparency. The authors started to analyze the case of a one-sided arbitrary termination [15].

Firstly, the present article aims to finish the analysis and measurements, by considering either-end arbitrary loads. Secondly, this article uses novel geometric reasoning to find a transparent strict upper bound.

\section{Article Structure}

Op 't Land's existing modified Taylor model for fieldto-trace coupling with characteristic terminal loads will be recalled in Section II. Building on top of that, the more general case of arbitrary loads at both terminals will be studied in Section III to extend the existing model. The resulting, accurate but opaque model will then be approximated to become pessimistic but transparent in Section IV. In order to gain confidence in both the accurate and the pessimistic models, they will both be compared to measurement in Section V. Based on these results and on actual model limitations, conclusions and recommendations for future research will be given in Section VI.

\section{Characteristic LoAdS}

The coupling of a far field to a bifilar, electrically short line will first be considered. Then, the line will be made electrically long. Finally, the case of a multi-segment microstrip trace will be considered. This development is a quick summary of the author's earlier work, a mathematical derivation can be found in [14] and an intuitive explanation in [15].

Consider a far field illumination impinging on a bifilar, lossless transmission line of length $\ell$, terminated at both sides in its characteristic impedance (Figure 2a). According to Taylor's formulation [3], the line needs to be meshed in small slices $\Delta z$, such that the illuminating field can be considered uniform along each slice. Consequently, for an electrically 
short line (or low frequencies), a single cell of $\Delta z=\ell$ suffices. The induced terminal voltages can be found by inspecting Figure $2 \mathrm{~b}$ and neglecting the transmission line:

$$
V_{\mathrm{LF}}=-\frac{1}{2} \mathrm{j} \omega c E_{t} Z_{c} h \ell \mp \frac{1}{2} \mathrm{j} \omega \mu_{0} H_{n} h \ell,
$$

where $c$ is the per-unit-length (pul) capacitance of the line. Unless otherwise noted, the near-end and far-end results are presented simultaneously throughout the paper; e.g. $\mp$ means minus for the near end and plus for the far end.

For an electrically long line (or high frequencies), the field is no longer uniform along the line, so more slices should be used, causing the solution to be no longer closed-form. Alternatively, the low-frequency solution of (3) can be corrected with a factor $K$ for long-line effects.

Consider the far-end induced voltage under endfire illumination $\left(E=E_{t}, H=H_{n}\right.$ and $\left.k_{p}=\omega / \mathrm{c}_{0}\right)$. Because of the characteristic terminations, there are no reflections on the line and only the forward-going eigenwave needs to be considered. Notice that although the endfire illumination and the forwardgoing eigenwave are no longer spatially uniform, they are going in the same direction at the same speed. Consequently, the illumination and the eigenwave have the same phase at every position $z$. Therefore, the far-end induced voltage still is that of (3).

Now imagine that the line slows down waves to phase speed $v$ (the wave number becomes $\beta=\omega / v$ ). The normalized eigenwave amplitude $w$ is just a phasor:

$$
w(z)=\mathrm{e}^{ \pm \mathrm{j} \beta z},
$$

where $z$ is the position on the line with respect to the near end. Similarly, the illuminating wave has normalized amplitude $i$ :

$$
i(z)=\mathrm{e}^{-\mathrm{j} k_{p} z} .
$$

At the near end $(z=0)$, the illuminating wave and the eigenwave are in phase, so the slice $\mathrm{d} z$ will contribute as in



(a) Line geometry: subscripts $t, n$ and $p$ (or, for this segment, $x, y$ and $z$ ) denote endfire excitation field components, respectively transversal, normal and parallel to the line segment. $Z_{c}$ denotes the line's characteristic impedance.

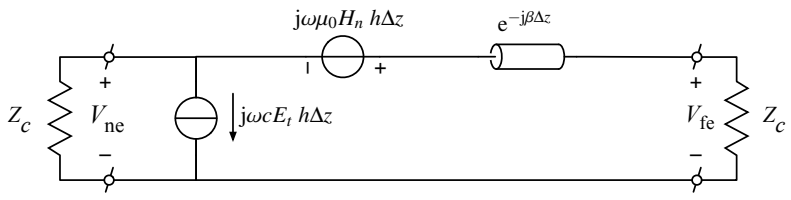

(b) Taylor's cell: approximation of passive transmission line slice $\Delta z$, with a voltage source representing the electromotive force (emf) and current source representing the electrostatic force (electric induction). $c$ denotes the per-unitlength (PUL) capacity of the line, $\beta$ is line's wavenumber.

Figure 2. Modeling the coupling of an electromagnetic wave to a two-wire transmission line. $+(\mathrm{d} z / \ell) V_{\mathrm{LF}}$. Little by little, they run out of phase; imagine a position $z$ where both waves are in opposition of phase. For this slice $\mathrm{d} z$, the contribution will be opposite: $-(\mathrm{d} z / \ell) V_{\mathrm{LF}}$. Integrating these contribution factors along the line gives the cross-correlation:

$$
K=\frac{1}{\ell} \int_{0}^{\ell} i(z) \cdot w^{*}(z) \mathrm{d} z=\frac{1}{\mathrm{j}\left(-k_{p} \mp \beta\right) \ell}\left(\mathrm{e}^{\mathrm{j}\left(-k_{p} \mp \beta\right) \ell}-1\right) .
$$

The either-end terminal voltages are now simply

$$
\begin{aligned}
& V_{\text {ne }}=K_{\text {ne }} V_{\mathrm{LF}, n \mathrm{e}} \\
& V_{\mathrm{fe}}=K_{\mathrm{fe}} V_{\mathrm{LF}, \mathrm{fe}} \mathrm{e}^{-\mathrm{j} \beta \ell},
\end{aligned}
$$

which holds for any angle of incidence and polarization. The $\mathrm{e}^{-\mathrm{j} \beta \ell}$ serves to move the phase reference to the far end.

Practical PCB traces are more complicated than a bifilar transmission line in vacuum. For simplicity, only a finite series of straight microstrip segments of equal width, with negligible parasitics at the transitions over an infinite ground plane, illuminated by a grazing incident, vertically polarized plane wave will be considered, as shown in Figure 3.

Under these conditions the field in the substrate is like those shown in Figure 4: both field components are doubled because of the ground plane, and the electric field is weaker because of the substrate's permittivity. The wave in the substrate needs to follow that imposed in free space, so the wave vector is the same.

The coupling to $N$ straight line segments can be found by superposition, because the segments are matched. That is, for each segment $u$, the contribution to the low-frequency induced terminal voltages $V_{\mathrm{LF}}^{u}$ needs to be calculated, multiplied with the correction factor $K^{u}$ :

$$
\begin{aligned}
& V_{\mathrm{ne}}=\sum_{u=1}^{N} K_{\mathrm{ne}}^{u} V_{\mathrm{LF}, \mathrm{ne}}^{u} \\
& V_{\mathrm{fe}}=\sum_{u=1}^{N} K_{\mathrm{fe}}^{u} V_{\mathrm{LF}, \mathrm{fe}}^{u} \mathrm{e}^{-\mathrm{j} \beta \ell},
\end{aligned}
$$

where $V_{\mathrm{LF}, \mathrm{fe}}^{u}\left(V_{\mathrm{LF}, \mathrm{ne}}^{u}\right)$ denotes the low-frequency far-end (nearend) voltage of the $u$ th segment according to (3), taking into account the field orientation with respect to each line segment. $\ell$ is a shorthand for the line's total length $\sum_{u=1}^{N} \ell_{u} . K_{\mathrm{fe}}^{u}$ is the far-end correction factor of the $u$ th line segment, which also needs to take into account the relative orientation of the field. It can be shown that

$$
K^{u}=\frac{1}{\mathrm{j}\left(-k_{s} \mp \beta\right) \ell_{u}}\left(\mathrm{e}^{\mathrm{j}\left(-k_{r} r_{\text {end }} \mp \beta s_{\text {end }}\right)}-\mathrm{e}^{\mathrm{j}\left(-k_{r} r_{\text {begin }} \mp \beta s_{\text {begin }}\right)}\right),
$$

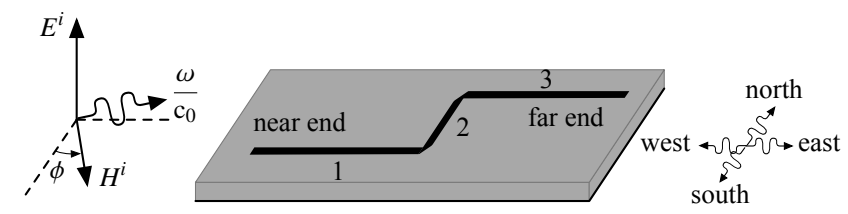

Figure 3. Example of the class of PCB traces considered in this paper: this particular trace has 3 segments. 
where $s$ denotes length along the line segment (distance from the near end) and $r$ denotes length in the propagation direction of the illuminating wave. $k_{s}$ is the incident wave vector along the line segment and $k_{r}$ is the incident wave number.

\section{MisMATCHED LOADS}

Basic transmission line theory will now be applied to obtain the either-end induced voltages for arbitrary (generally mismatched) loads.

From the foregoing Section II, $V_{\text {ne }}$ and $V_{\mathrm{fe}}$ are known, which can be considered voltage waves coming out of a transmission line of characteristic impedance $Z_{c}$. These voltage waves will then generally be reflected by the either-end terminal impedances $Z_{\mathrm{ee}}$ according to their voltage reflection coefficients:

$$
\Gamma_{\mathrm{ee}}=\frac{Z_{\mathrm{ee}}-Z_{c}}{Z_{\mathrm{ee}}+Z_{c}} .
$$

As illustrated in Figure 5, the far-end induced voltage wave $V_{\text {fe }}$ will be reflected by the far-end terminal impedance, then delayed by the transmission line. Together with the near-end induced voltage $V_{\text {ne }}$, it can be considered a voltage wave $V_{\text {inc,ne }}$ incident on the near-end terminal impedance:

$$
V_{\text {inc,ne }}=V_{\text {ne }}+\Gamma_{\mathrm{fe}} V_{\mathrm{fe}} \mathrm{e}^{-\mathrm{j} \beta \ell} .
$$

If the near-end would be characteristically terminated $\left(Z_{\mathrm{ne}}=\right.$ $Z_{c}$ ), this is exactly the final near-end voltage [15].

In general, however, a near-end reflection will occur:

$$
V_{\text {refl,ne }}=\Gamma_{\text {ne }} V_{\text {inc,ne }}=\frac{Z_{\text {ne }}-Z_{c}}{Z_{\text {ne }}+Z_{c}} V_{\text {inc,ne }}
$$

that is, the near-end reflected wave would induce a voltage $V_{\text {refl,ne }}$ on a characteristic load. With this knowledge, the reflected wave can also be represented as an equivalent nearend voltage source as shown in Figure 6a:

$$
V_{\text {refl,ne,equiv }}=V_{\text {refl,ne }} \frac{Z_{c}+Z_{\text {ne }}}{Z_{c}}=V_{\text {inc,ne }} \frac{Z_{\text {ne }}-Z_{c}}{Z_{\text {ne }}+Z_{c}} \frac{Z_{e}+Z_{\text {ne }}}{Z_{c}}
$$
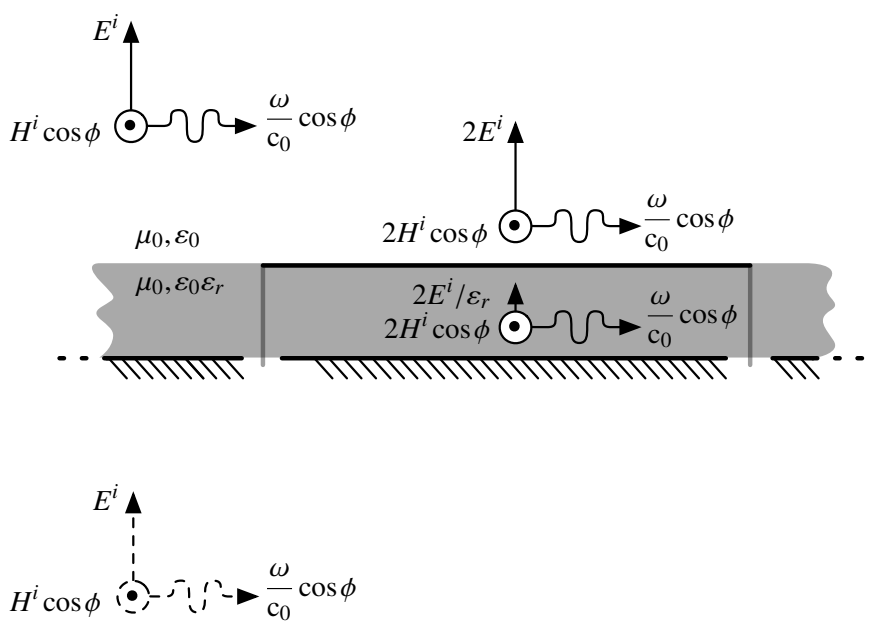

Figure 4. The far-away plane wave source (top left) is reflected by the microstrip's ground plane (image source at the bottom left). This results in the fields shown at the right.
To avoid having to consider infinite reflections going up and down the line, the far-end terminal impedance is translated to the near end:

$$
\begin{aligned}
& \Gamma_{\mathrm{fe} @ \mathrm{ne}}=\mathrm{e}^{-2 \mathrm{j} \beta \ell} \Gamma_{\mathrm{fe}} \\
& Z_{\mathrm{fe} @ \mathrm{ne}}=Z_{c} \frac{1+\Gamma_{\mathrm{fe} @ \mathrm{ne}}}{1-\Gamma_{\mathrm{fe} @ \mathrm{ne}}},
\end{aligned}
$$

where '@ne' denotes 'as seen at the near end'. Now, the final near-end reflected voltage can be calculated using the voltage divider of Figure 6b:

$$
V_{\text {refl,ne,final }}=V_{\text {refl,ne,equiv }} \frac{Z_{\mathrm{fe} @ \text { ne }}}{Z_{\mathrm{ne}}+Z_{\mathrm{fe} @ \mathrm{ne}}} .
$$

The total near-end voltage is the sum of the incident and the final reflected voltage:

$$
\begin{aligned}
V_{\text {ne,final }} & =V_{\text {inc,ne }}+V_{\text {refl,ne,final }} \\
& =\left(V_{\text {ne }}+\Gamma_{\mathrm{fe}} V_{\mathrm{fe}} \mathrm{e}^{-\mathrm{j} \beta \ell}\right)\left(1+\frac{Z_{\mathrm{ne}}-Z_{c}}{Z_{c}} \frac{Z_{\mathrm{fe} @ \mathrm{ne}}}{Z_{\mathrm{ne}}+Z_{\mathrm{fe} @ \mathrm{ne}}}\right),
\end{aligned}
$$

which also holds, mutatis mutandis, for the total far-end voltage. Note that this solution is, albeit lengthy, closed-form.

\section{WORST-CASE CONCLUSIONS}

The conclusion of (19), especially when expanded, is very opaque. In order to gain insight, a design-oriented approximation will now be attempted.

Particularly, an EMC-aware PCB designer will try to achieve sufficient immunity, having some influence on routing and terminal impedances. Sufficient immunity is achieved when worst-case (maximum) voltages at the trace terminals do not exceed an immunity threshold that depends on the connected components. Routing influence may consist in reducing trace length or changing trace width within the practical possibilities. Terminal impedances may also be tuned, by introducing frequency-dependent parallel or series impedances.

Especially for low-speed digital circuits, both terminal impedances are expected to be rather mismatched (i.e. some $\Omega$ connected to some $\mathrm{k} \Omega$ ). Furthermore, the engineer will be interested in the worst-case voltage magnitude over angle of incidence and frequency.

To deal with the complexity of (19), its terms and factors will be separately inspected to find an upper bound to them. The sum and product of these upper bounds will then constitute a generally loose upper bound to the entire expression.

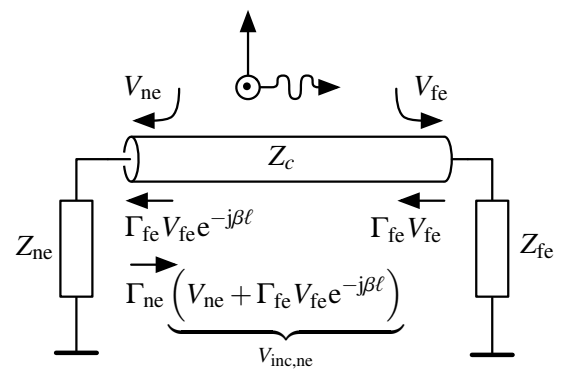

Figure 5. From top to bottom: the either-end induced voltage waves, the far-end reflection and the near-end reflection. 


\section{A. Multiple segments with characteristic loads}

Equation 19 starts with $V_{\text {ne }}$ and $V_{\text {fe }}$ : the near- and farend induced voltages when both ends are characteristically terminated. The transparent upper bound of [14] for one trace segment will now be recalled and extended to multiple segments.

For low frequencies, the worst-case voltage induced in a single trace segment occurs for end-fire excitation and at the near end [9]. Plugging the field strengths of Figure 4 into (3) and using the impedance of free space to write $H^{i}$ in terms of $E^{i}$, it amounts to [14]:

$$
\max \left|V_{\mathrm{LF}}\right|=\frac{\omega}{\mathrm{c}_{0}} E^{i} h \ell\left(1+\frac{\sqrt{\varepsilon_{r, \mathrm{eff}}}}{\varepsilon_{r}}\right),
$$

where $h$ is the substrate thickness, $\ell$ is the segment length and $\varepsilon_{r, \text { eff }}$ is the effective permittivity of the microstrip line: the average permittivity experienced by the electric field, so $1<\varepsilon_{r, \text { eff }}<\varepsilon_{r}$.

For high frequencies, the worst-case voltage induced in a single segment for grazing incidence occurs at the far-end and amounts to [14]:

$$
\max \left|V_{\mathrm{HF}}\right|=2 E^{i} h \frac{1-\frac{\sqrt{\varepsilon_{r, \mathrm{eff}}}}{\varepsilon_{r}}}{\sqrt{\varepsilon_{r, \mathrm{eff}}}-1},
$$

which is frequency- and length (!) independent.

Joining these two asymptotes gives a transparent worstcase envelope for the field-induced voltage in a single trace segment [14]. In the very worst case of a multi-segment trace, these envelopes add up in phase. Therefore, a loose upper bound to the either-end induced voltage $V_{\text {ee }}$ can be found by summing up the envelopes of each straight line segment:

$$
\max \left|V_{\mathrm{ee}}\right|=E^{i} h \min \{\underbrace{\frac{\omega}{\mathrm{c}_{0}} \ell\left(1+\frac{\sqrt{\varepsilon_{r, \mathrm{eff}}}}{\varepsilon_{r}}\right)}_{\text {low frequency }}, \underbrace{2 n \frac{1-\frac{\sqrt{\varepsilon_{r, \mathrm{eff}}}}{\varepsilon_{r}}}{\sqrt{\varepsilon_{r, \mathrm{eff}}}-1}}_{\text {high frequency }}\},
$$

where $\ell$ is the total length of the line and $n$ is the number of segments.

\section{B. Far-end Contribution}

Although the near- and far-end will never simultaneously amount to this upper bound, this pessimistic assumption simplifies the analysis. Moreover, the phasor $\mathrm{e}^{-\mathrm{j} \beta \ell}$ rotates with

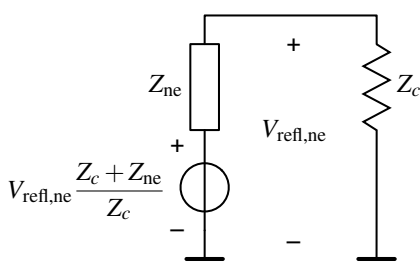

(a) First step: find the near-end voltage source equivalent to the reflected voltage wave, using a characteristic load.

Figure 6. Calculating the final effect of the near-end reflection.

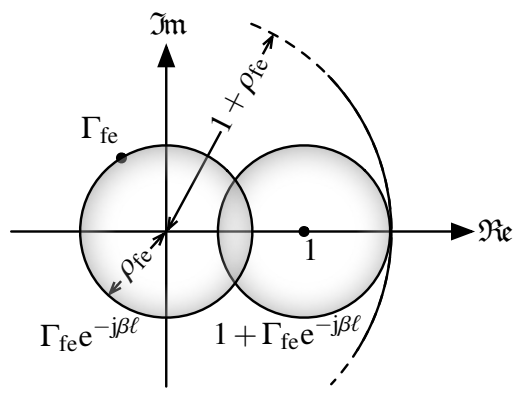

Figure 7. Geometric construction of (23), normalized by $\max \left|V_{\text {ee }}\right|$.

frequency, so in the worst case, the near- and far-end induced voltages will add up in phase. Therefore, the first factor of (19) can be safely approximated like so:

$$
\max \left|V_{\mathrm{ne}}+\Gamma_{\mathrm{fe}} V_{\mathrm{fe}} \mathrm{e}^{-\mathrm{j} \beta \ell}\right| \leq \max \left|V_{\mathrm{ee}}\right|\left(1+\rho_{\mathrm{fe}}\right),
$$

where $\rho_{\mathrm{fe}}$ is the magnitude of the far-end reflection coefficient $\Gamma_{\mathrm{fe}}$. This reasoning step is illustrated in Figure 7; notice that $\Gamma_{\text {fe } @ \text { ne }}$ describes a circle.

\section{Far-end Admittance Dependency}

The remaining opaque term of (19) is the last fraction: the goal is to find its maximum magnitude while $\mathrm{e}^{-\mathrm{j} \beta \ell}$ describes the unit circle (i.e. the product $\beta \ell$ takes all possible values). To that end, the fraction will be rearranged to obtain a difference, which has an obvious geometric interpretation on the complex plane: a distance.

$$
\begin{array}{r}
\max _{\beta \ell}\left|\frac{Z_{\mathrm{fe} @ \mathrm{ne}}}{Z_{\mathrm{fe} @ \mathrm{ne}}+Z_{\mathrm{ne}}}\right|=\max _{\beta \ell} \frac{1}{\mid 1+\frac{Z_{\mathrm{ne}}}{Z_{\mathrm{fe}} @ \mathrm{ne}}} \mid \\
=\frac{1}{\left|Z_{\mathrm{ne}}\right| \min _{\beta \ell}\left|\frac{1}{Z_{\mathrm{ne}}}+\frac{1}{Z_{\mathrm{fe} @ \mathrm{ne}}}\right|}=\frac{1}{\left|Z_{\mathrm{ne}}\right| \min _{\beta \ell}\left|-\frac{1}{Z_{\mathrm{ne}}}-\frac{1}{Z_{\mathrm{fe} @ \mathrm{ne}}}\right|} .
\end{array}
$$

Put geometrically: the worst case occurs when the mirrored near-end admittance $-Y_{\text {ne }}$ and the far-end admittance seen at the near end $Y_{\mathrm{fe}} @$ ne are closest. Now what does $Y_{\mathrm{fe} @ \text { ne }}$ look like, on the complex admittance plane?

Recall that admittance and reflection coefficient are related by:

$$
Y=\frac{1}{Z_{c}} \frac{1-\Gamma}{1+\Gamma}
$$

which is a Möbius transformation, so generalized circles in

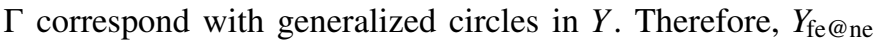
must be a circle. Moreover, this transformation is mirror symmetric about the real axis. As the $\Gamma_{\text {fe@ne }}$ itself is mirror symmetric about the real axis, its transform $Y_{\mathrm{fe} @ \text { ne }}$ must be mirror symmetric about the real axis too. Hence, the transform $Y_{\mathrm{fe} @ \text { ne }}$ must be a circle with a real center.

Now it suffices to find two transformed points to entirely define the transformed circle. Let us take the rightmost and the leftmost value of $\Gamma_{\mathrm{fe}} @$ ne, that is $\pm \rho_{\mathrm{fe}}$. Their admittance counterparts are

$$
Y_{\mathrm{fe} @ \mathrm{ne}}=\frac{1}{Z_{c}} \frac{1 \mp \rho_{\mathrm{fe}}}{1 \pm \rho_{\mathrm{fe}}} .
$$




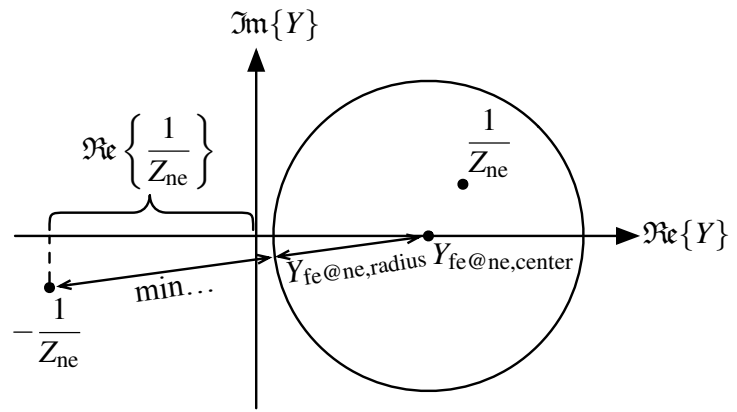

Figure 8. Geometric construction of (29). $Y_{\text {ne }}$ accidentally lies inside the $Y_{\mathrm{fe}} @$ ne circle.

As these points lie on a circle with a real center, the center and radius can be found by taking half the sum and half the difference:

$$
\begin{aligned}
& Y_{\mathrm{fe} @ \text { ne,radius }}=\frac{1}{2} \frac{1}{Z_{c}}\left(\frac{1+\rho_{\mathrm{fe}}}{1-\rho_{\mathrm{fe}}}-\frac{1-\rho_{\mathrm{fe}}}{1+\rho_{\mathrm{fe}}}\right)=\frac{1}{Z_{c}} \frac{2 \rho_{\mathrm{fe}}}{1-\rho_{\mathrm{fe}}^{2}} \\
& Y_{\mathrm{fe} @ \text { ne,center }}=\frac{1}{2} \frac{1}{Z_{c}}\left(\frac{1+\rho_{\mathrm{fe}}}{1-\rho_{\mathrm{fe}}}+\frac{1-\rho_{\mathrm{fe}}}{1+\rho_{\mathrm{fe}}}\right)=\frac{1}{Z_{c}} \frac{1+\rho_{\mathrm{fe}}^{2}}{1-\rho_{\mathrm{fe}}^{2}}
\end{aligned}
$$

The sought minimum is constructed in Figure 8: $Y_{\mathrm{fe} @ \mathrm{ne}}$ describes a circle and $-1 / Z_{\mathrm{ne}}$ always lies in the left half plane. The minimum distance between a circle and a point is the distance from the circle's center to the point minus the circle's radius. For a rather mismatched far-end load (a circle close to the imaginary axis), a safe approximation can be found by inspection of Figure 8:

$$
\min _{\beta \ell}\left|-\frac{1}{Z_{\mathrm{ne}}}-\frac{1}{Z_{\mathrm{fe}} @ \mathrm{ne}}\right| \geq \mathfrak{R}\left\{\frac{1}{Z_{\mathrm{ne}}}\right\} .
$$

Note that other approximations are possible, but for the sake of transparency, this candidate was retained.

Plugging this result into (24) yields

$$
\max _{\beta \ell}\left|\frac{Z_{\mathrm{fe} @ \mathrm{ne}}}{Z_{\mathrm{fe} @ \mathrm{ne}}+Z_{\mathrm{ne}}}\right| \leq \frac{1}{\left|Z_{\mathrm{ne}}\right| \frac{1}{\Re\left(Z_{\mathrm{ne}}\right\}}}=\frac{1}{\left|Z_{\mathrm{ne}}\right| \frac{\Re \mathfrak{k}\left\{Z_{\mathrm{ne}}\right\}}{\left|Z_{\mathrm{ne}}\right|^{\mid}}}=\frac{1}{\cos \angle Z_{\mathrm{ne}}} .
$$

\section{Upper Bound}

Plugging the worst case terms and factors in (19), this upper bound is obtained:

$$
V_{\text {ne,final }} \leq \max \mid V_{\text {ee }}\left(1+\rho_{\mathrm{fe}}\right)\left(1+\left|\frac{Z_{\mathrm{ne}}}{Z_{c}}-1\right| \frac{1}{\cos \angle Z_{\mathrm{ne}}}\right),
$$

mutatis mutandis for the far end.

Note that this upper bound is particularly pessimistic for rather matched far-end loads. Stricter upper bounds exist and were found, but turned out to be opaque.

One well-known best design practice is confirmed by this result: matching helps. This can be seen from (31), because the smaller $\rho_{\mathrm{fe}}$ and the closer $Z_{\mathrm{ne}}$ to $Z_{c}$, the smaller the upper bound. The last factor confirms that a purely capacitive (inductive) near-end load is particularly bad, and adding a small resistance in series (parallel) may dramatically reduce resonances. However, PCB designers should bear in mind that matching may also be improved for free (just by changing the trace width).

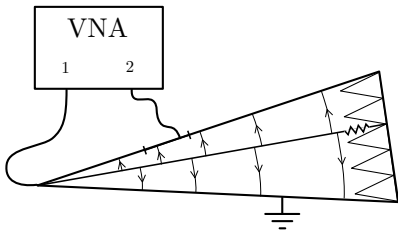

Figure 9. Cross section of the GTEM cell used to illuminated PCBs: port 1 of the VNA is connected to the GTEM cell input, port 2 is connected to one terminal of the measured trace.

\section{Measurements}

Now, above results will be challenged by measurements and full-wave simulations. First, the upper bound for a multisegment trace with characteristic loads of Section IV-A will be gauged using GTEM cell measurements. Then, the accurate but opaque model of Section III will be compared with measurement and full-wave simulation of an either-end mismatched trace. Finally, the transparent upper bound for a multi-segment trace with arbitrary loads of Section IV-D will be gauged by measurements.

\section{A. Upper Bound for a Matched Multi-segment Trace}

To challenge the upper bound on (22) of the coupling to a multi-segment trace as shown in Figure 3, an illumination and a trace is needed.

To illuminate with a horizontally polarized, grazing incident plane wave, a Gigahertz Transverse Electromagnetic (GTEM) cell is used. A GTEM cell is a rectangular, paraxial, tapered $50 \Omega$ waveguide. The narrow end of the taper is equipped with a coaxial connector and the wide end is terminated with a hybrid resistive/absorber $50 \Omega$ load. The center conductor is called the septum. The Schaeffner 250A-SAE GTEM cell used in this paper sports a top opening, where a $10 \times 10 \mathrm{~cm}$ PCB can be placed. When connected to a Vector Network Analyzer (VNA) as shown in Figure 9, the field between the PCB and septum can be considered the air field shown in Figure 4:

$$
2 E^{i}=\frac{V_{\text {septum }}}{d},
$$

where $d$ is the average distance between the septum and the PCB's ground plane. The measured $S_{21}$ parameter equals the voltage transfer between GTEM septum and a $50 \Omega$ trace terminal. Therefore, from here on out, the calculations will use the incident electric field generated by $1 \mathrm{~V}$ at the septum.

The trace has three orthogonal segments, like the trace of Figure 3, having lengths of respectively $25 \mathrm{~mm}, 16.7 \mathrm{~mm}$ and $25 \mathrm{~mm}$. The trace is a $50 \Omega$ microstrip on the outer layer of a four-layer industrial stack-up. The substrate between the outer layer and the ground plane just below has a relative permittivity $\varepsilon_{r}$ of 4.57 and a thickness $h$ of $362 \mu \mathrm{m}$. Both terminals are equipped with SMA connectors, giving access from outside the GTEM cell. All transitions were matched to $50 \Omega$. More details on the precise stack up in [15].

The described set-up was realized and the $S_{21}$ parameter was measured to one end of the trace, while terminating the other in $50 \Omega$. The square PCB was rotated in order to illuminate 


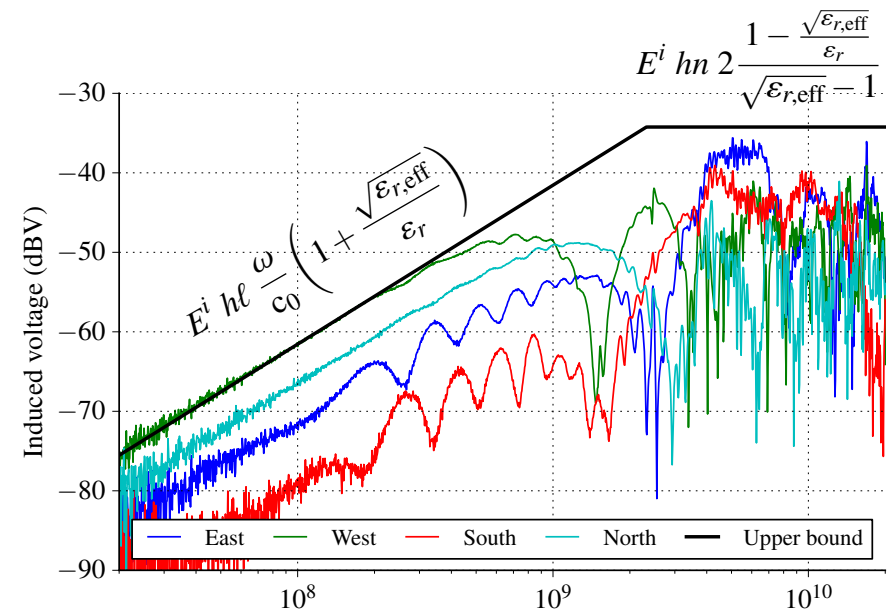

Figure 10. Measured $S_{21}$ of the multi-segment trace in four positions (port 1 is the GTEM cell input, port 2 is one trace end, the other being characteristically terminated), compared to the transparent upper bound of (22), calculated with $2 E^{i}=23.8 \mathrm{~V} / \mathrm{m}$ (the GTEM field with $1 \mathrm{~V}$ at it's input).

the trace from all four cardinal directions. The results are compared to the upper bound of (22) in Figure 10.

The upper bound seems to envelope all measurements, as expected. However, even the western illuminated trace should be about $2 \mathrm{~dB}$ below the low-frequency asymptote, because there is no magnetic coupling to the second segment. Fullwave simulations (not shown here) yield the expected curves, $2 \mathrm{~dB}$ lower than the experiments. Despite that, the upper bound is rather tight, even when taking into account this error.

\section{B. Accurate Model of a Mismatched Multi-segment Trace}

To assess the correctness of the accurate model for coupling to a multi-segment trace with either-end mismatched loads, suitable loads need to be connected.

At the far end, an Agilent 85052D-60006 short circuit, having a delay of $31.78 \mathrm{ps}$, was connected to the SMA connector. Including the SMA connector and the PCB, the farend impedance is modeled as a perfect short circuit, delayed by a $50 \Omega$, lossless transmission line with a 79.95 ps delay [15].

At the near end, a mismatched load is needed that also allows to measure the voltage with a VNA. For that purpose, the trace was cut open at the near end and a surface mount $220 \Omega$ resistor was soldered in series. Just before, this resistor was characterized up to $20 \mathrm{GHz}$ with a low-budget fixture [16]. The measured impedance could be satisfactorily modeled by a $44 \mathrm{fF}$ parasitic capacitance. As the VNA, connector and transition are matched to $50 \Omega$, they can be modeled as a simple $50 \Omega$ load. The resulting model is shown in Figure 11 . To estimate the final near-end voltage, the $S_{21}$ readout of the VNA is divided by the voltage transfer of the resistive voltage divider.

Equation 19 is evaluated using a Python script, calling upon scikit-rf to perform transmission-line calculations like (16) and (17). To distinguish between fundamental model errors and measurement non-idealities, a full-wave simulation is run

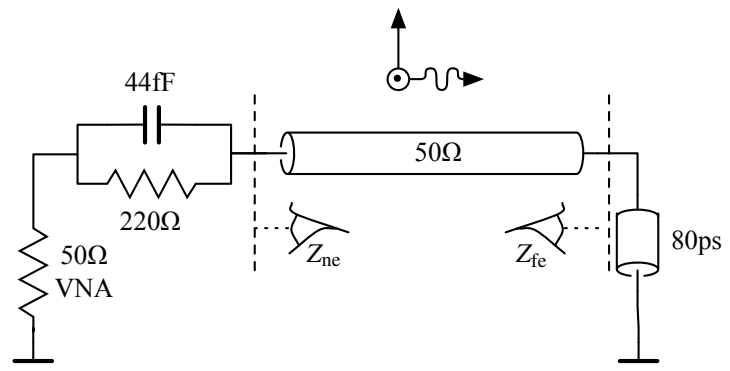

Figure 11. Near-end and far-end load models. The near-end resistive divider presents a mismatched load, yet allowing to estimate the final near-end induced voltage.

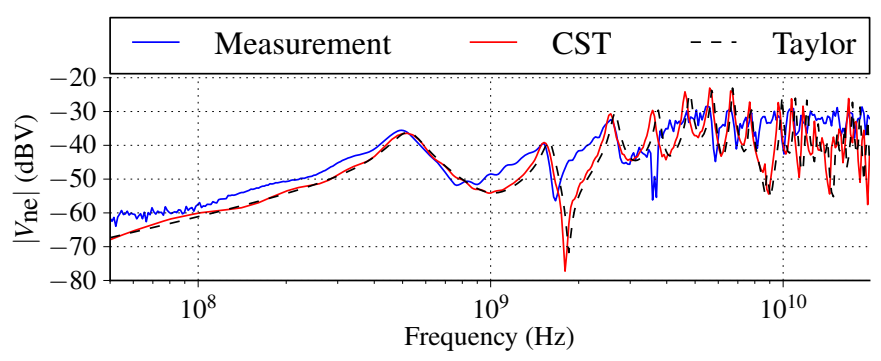

(a) Worst correlation: endfire illumination from the near-end side.

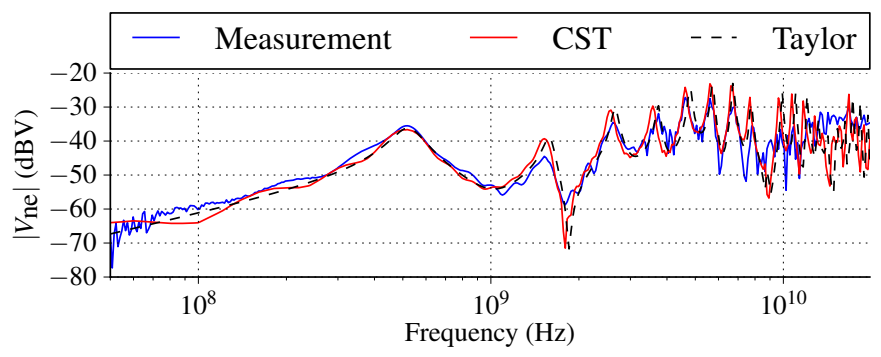

(b) Best correlation: endfire illumination from the far-end side.

Figure 12. Induced voltage in the near end of the multi-segment trace, as measured (port 1 on the GTEM cell input, port 2 is the trace's near end equipped with a probe, the far end being shorted using a calibration standard), as simulated with the full-wave solver CST and as predicted with the accurate model of (19).

using CST. Measurement, full-wave simulation and Taylor calculation are repeated for the other cardinal directions.

The log-frequency weighted average difference between measurement and Taylor model, averaged over all four cardinal directions is $-1.8 \mathrm{~dB}$. The average difference between fullwave simulation and Taylor model is $-0.3 \mathrm{~dB}$. The directions with the worst and the best measurement correlation are shown in Figure 12a and 12b, respectively.

\section{Upper Bound for a Mismatched Multi-segment Trace}

As can be seen in Figure 13, the measured coupling to the trace in all four cardinal directions indeed remains below the transparent upper bound of (31). It can be noticed that, for this particular trace and these four directions of illumination, the proposed upper bound is $7 \mathrm{~dB}$ too pessimistic for the low frequency asymptote, and $13 \mathrm{~dB}$ too pessimistic for the high frequency asymptote. 


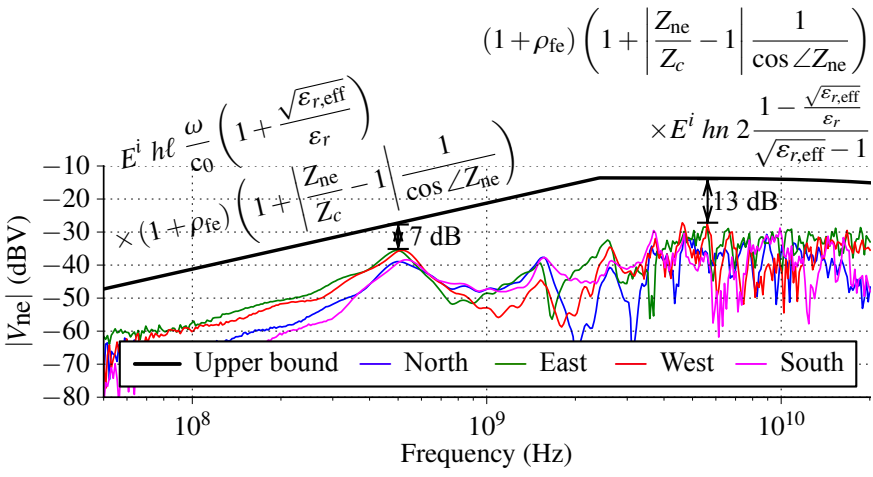

Figure 13. Measured voltage at the near end of the multi-segment trace in all cardinal positions (port 1 is the GTEM cell input, port 2 is the trace's near end equipped with a voltage divider, the far end being shorted), compared to the transparent upper bound of (31). The high-frequency limit is not flat, because of the parasitic capacitance in the near-end load.

\section{CONCLUSIONS}

Firstly, the existing modified Taylor model for the coupling of a vertically polarized, grazing-incident plane wave to a multi-segment, uniform microstrip trace with matched loads was extended to comprise arbitrary terminal loads: (19). This is an opaque, but closed-form solution. It was applied to predict the coupling to a three-segment PCB trace with eitherend mismatched loads. Fair agreement with a GTEM cell measurement was obtained: $-1.8 \mathrm{~dB}$ average error and good agreement with a full-wave CST simulation: $-0.3 \mathrm{~dB}$.

The extended Taylor model for mismatched terminal loads is based on the assumptions of weak coupling and a TEM transmission line. Because CST yields about the same results without relying on these assumptions, it can be concluded that for this case study, weak coupling and TEM assumptions hold up to about $20 \mathrm{GHz}$. The GTEM cell emulates a plane wave, but due to standing waves and parasitic modes, the illumination is more complicated than that. Moreover, the illuminated trace has losses and the $220 \Omega$ probe resistor is illuminated itself, too. All these non-idealities are neither taken into account by the full-wave simulation nor by the extended Taylor model. Therefore, they might explain the discrepancies between measurement and extended Taylor model.

Secondly, this opaque solution was approximated to obtain a transparent upper bound for multi-segment traces with rather mismatched terminal loads, as shown in Figure 13. The upper bound consists of the existing upper bound for matched loads and a factor that accounts for the either-end mismatch. Indeed, this upper bound was not surpassed by the mentioned GTEM cell measurements.

In future research, the illumination should be generalized to arbitrarily-polarized, arbitrarily-incident plane waves, because in reality, perturbing electromagnetic fields are unknown. Considering the worst case may help to keep the number of variables reasonable.

As for the terminal loads, an industrial use case should prove whether or not the upper bound developed in this paper is useful. Moreover, as the authors have only started to enjoy Möbius transformations, stricter yet transparent upper bounds may have been overlooked.

\section{ACKNOWLEDGEMENTS}

The authors are indebted to Jean Sudolski for the fruitful idea of a distance on the admittance plane. This research project was co-financed by the French national project SEISME (simulation of emissions and immunity of electronic systems).

\section{REFERENCES}

[1] R. D. Middlebrook, Design-Oriented Analysis Rules and Tools. Ardem Associates, 2007. [Online]. Available: http://www.RDMiddlebrook. com

[2] C. A. Nucci, F. Rachidi, and M. Rubinstein, "An overview of field-totransmission line interaction", Applied Computational Electromagnetics Society Newsletter, vol. 22, no. 1, pp. 9-27, 2007.

[3] C. D. Taylor, R. Satterwhite, and C. W. Harrison Jr., "The response of a terminated two-wire transmission line excited by a nonuniform electromagnetic field", Antennas and Propagation, IEEE Transactions on, vol. 13, no. 6, pp. 987-989, Nov. 1965. DOI: 10.1109/TAP.1965. 1138574.

[4] A. K. Agrawal, H. J. Price, and S. H. Gurbaxani, "Transient response of multiconductor transmission lines excited by a nonuniform electromagnetic field", Electromagnetic Compatibility, IEEE Transactions on, vol. EMC-22, no. 2, pp. 119-129, May 1980. DOI: 10.1109/TEMC. 1980.303824.

[5] F. Rachidi, "Formulation of the field-to-transmission line coupling equations in terms of magnetic excitation field", Electromagnetic Compatibility, IEEE Transactions on, vol. 35, no. 3, pp. 404-407, Aug. 1993. DOI: 10.1109/15.277316.

[6] C. Baum, T. Liu, and F. Tesche, "On the analysis of general multiconductor transmission-line networks", Interaction Note, vol. 350, pp. 467-547, 1978.

[7] C. R. Paul, Analysis of multiconductor transmission lines. WileyInterscience, 2007.

[8] S. Op 't Land, "Equivalence check of Op 't Land's and Paul's field-to-line coupling solutions", ESEO-EMC, Technical Report, 2016. [Online]. Available: https://hal.archives-ouvertes.fr/hal-01265116.

[9] M. Leone and H. L. Singer, "On the coupling of an external electromagnetic field to a printed circuit board trace", Electromagnetic Compatibility, IEEE Transactions on, vol. 41, no. 4, pp. 418-424, Nov. 1999. DOI: $10.1109 / 15.809842$.

[10] J. L. Lagos and F. L. Fiori, "Worst-case induced disturbances in digital and analog interchip interconnects by an external electromagnetic plane wave - part I: Modeling and algorithm", Electromagnetic Compatibility, IEEE Transactions on, vol. 53, no. 1, pp. 178-184, Feb. 2011. DOI: 10.1109/TEMC.2010.2085005.

[11] F. Vanhee, D. Pissoort, J. Catrysse, G. Vandenbosch, and G. Gielen, "Efficient reciprocity-based algorithm to predict worst case induced disturbances on multiconductor transmission lines due to incoming plane waves", Electromagnetic Compatibility, IEEE Transactions on, vol. 55, no. 1, pp. 208-216, Feb. 2013. DOI: 10.1109/TEMC.2012. 2208754.

[12] M. Magdowski and R. Vick, "Closed-form formulas for the stochastic electromagnetic field coupling to a transmission line with arbitrary loads", Electromagnetic Compatibility, IEEE Transactions on, vol. 54, no. 5, pp. 1147-1152, Oct. 2012. DOI: 10.1109/TEMC.2012.2193130.

[13] M. Leone, "Radiated susceptibility on the printed-circuit-board level: Simulation and measurement", Electromagnetic Compatibility, IEEE Transactions on, vol. 47, no. 3, pp. 471-478, Aug. 2005. DOI: 10 . 1109/TEMC.2005.850682.

[14] S. T. Op 't Land, M. Ramdani, R. Perdriau, M. Leone, and M. Drissi, "Simple, Taylor-based worst-case model for field-to-line coupling", JPIER, vol. 140, pp. 297-311, Jun. 2013. DOI: 10.2528/ PIER13041207.

[15] S. T. Op 't Land, M. Ramdani, R. Perdriau, Y. Braux, and M. Drissi, "Using a modified Taylor cell to validate simulation and measurement of field-to-shorted-trace coupling", IEEE Transactions on Electromagnetic Compatibility, vol. 56, no. 4, pp. 864-870, 2014. DOI: 10.1109/ TEMC.2014.2313231.

[16] S. T. Op 't Land, R. Perdriau, and M. Ramdani, "Low-cost 0603 SMD impedance measurement fixture", Mediterranean Telecommunication Journal, vol. 3, no. 1, pp. 40-43, Feb. 2013, Presented earlier on Congrès Mediterranéen des Télécommunications et Exposition, 22 to 24 March 2012, Fez, Marocco. 